\title{
Accurate measurement of extremely low surface recombination velocities on charged, oxidized silicon surfaces using a simple metal-oxide-semiconductor structure
}

\author{
W. E. Jellett ${ }^{\mathrm{a})}$ and K. J. Weber \\ Centre for Sustainable Energy Systems, Faculty of Engineering and Information Technology, The Australian \\ National University, Canberra, ACT 0200, Australia
}

(Received 19 October 2006; accepted 20 December 2006; published online 24 January 2007)

\begin{abstract}
The authors report a simple technique to determine the surface recombination velocity of silicon and other semiconductor surfaces which have been passivated with a dielectric layer, as a function of charge density. A metal-oxide-semiconductor structure, employing large area, partially transparent metal contacts, is used to enable the charging of the surfaces. Simultaneous measurement of the emitter saturation current density $J_{\mathrm{oe}}$ and the effective instantaneous lifetime $\tau_{\text {inst }}$ allows accurate extraction of the effective surface recombination velocity $S_{\text {eff }}$ at any given injection level. Extremely low $J_{\mathrm{oe}}$ values of $1.8 \mathrm{fA} \mathrm{cm}^{-2}$ are measured on the silicon-silicon oxide $\left(\mathrm{Si}-\mathrm{SiO}_{2}\right)$ interface of a thermally oxidized, charged wafer. (C) 2007 American Institute of Physics.
\end{abstract}

[DOI: $10.1063 / 1.2434172]$

It is well known that the presence of electrical surface charge can have a dramatic effect on the recombination rates of photogenerated carriers at the surfaces of silicon $(\mathrm{Si})$ solar cells and other devices, as a result of band bending induced at the Si surface. On lightly to moderately doped silicon, the presence of a large amount of either positive or negative charge leads to low surface recombination rates corresponding to accumulation or strong inversion conditions at the surface, while the recombination rate reaches a maximum when the surface is in depletion, with approximately equal concentrations of electrons and holes. ${ }^{1,2}$ Even on diffused surfaces, the presence of charge can have a dramatic effect on the electrical interface properties. ${ }^{3}$ The majority of measurements of the effect of charge on surface recombination have been carried out on moderately doped silicon with a doping around $1 \Omega \mathrm{cm}$, since this type of material is most commonly used for photovoltaic applications. Charging the samples is most commonly done using a corona discharge chamber, since this method has the advantage of not interfering with the optical properties of the sample being measured. Accurate determination of the charge density requires separate measurement using a Kelvin probe. Alternative methods, such as the measurement of the photovoltage using mercury probes, together with the use of a transparent conductive oxide layer for sample charging, have also been used. ${ }^{4}$ While these measurements have clearly shown the qualitative variation of the surface recombination rate with charge density, accurate determination of surface recombination becomes difficult as the surface recombination rate approaches very low values.

In this letter we present minority carrier lifetime measurements on lightly doped, thermally oxidized $\mathrm{Si}$ wafers. The use of lightly doped $\left(10^{13}-10^{14} \mathrm{~cm}^{-3}\right)$ material has the significant advantage that it allows the accurate determination of the emitter saturation current density $J_{\mathrm{oe}}$. This parameter in turn can be used to obtain more accurate values of the

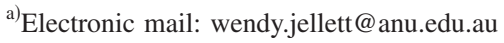

more commonly measured parameter, the effective surface recombination velocity $S_{\text {eff }}$.

Samples used for the experiments were float zone $[(100)] p$ type wafers with a B doping concentration of $\sim 10^{14} \mathrm{~cm}^{-3}$. Wafers were supplied lapped and etched. They were etched in hydrofluoric/nitric acid solution to remove $\sim 20 \mu \mathrm{m}$ from each surface, cleaved into quarters, and then given a standard RCA clean. ${ }^{5}$ A $50 \mathrm{~nm}$ thick thermal oxide was grown at $1000{ }^{\circ} \mathrm{C}$ for 60 min without trichloroethylene followed by a $\mathrm{N}_{2}$ annealing at the same temperature for $30 \mathrm{~min}$, and a forming gas annealing for $30 \mathrm{~min}$ at $400{ }^{\circ} \mathrm{C}$.

Using a shadow mask aluminium (Al) was evaporated over a circular area onto both sides of each quarter to a thickness of $\sim 5 \mathrm{~nm}$ to create a symmetric metal-oxidesemiconductor (MOS) structure. The diameter of the metallized area was at least $5 \mathrm{~mm}$ larger than the sensing area of the inductive coil for lifetime measurements in order to minimize edge effects. Contact to Al layers was made using conductive silver paste, while contact to the silicon bulk was made by removing a small amount of oxide in one of the corners of the sample and applying silver paste. The final structure is shown in Fig. 1. The wafer quarter was positioned so that the metallized region was centered over the inductive coil. The metal thickness was carefully chosen to ensure minimal interference with the lifetime measurement.

While the corona discharge is an accurate alternative technique for applying surface charge, a disadvantage of the method is that it requires the sample to be moved between the measurement equipment, the corona discharge chamber, and the Kelvin probe for each data point. In contrast, the use of a MOS structure allows all measurements to be carried out without moving the sample, thus minimizing the possibility of sample damage during handling, allowing more rapid measurements, and minimizing error introduced by sample movement, or the possible decay of charges between Kelvin probe and lifetime measurements.

Measurements were carried out using the inductively coupled photoconductivity decay technique. ${ }^{6,7}$ The effective lifetime is determined from the rate of decay of the wafer photoconductivity following the application of a light pulse. 


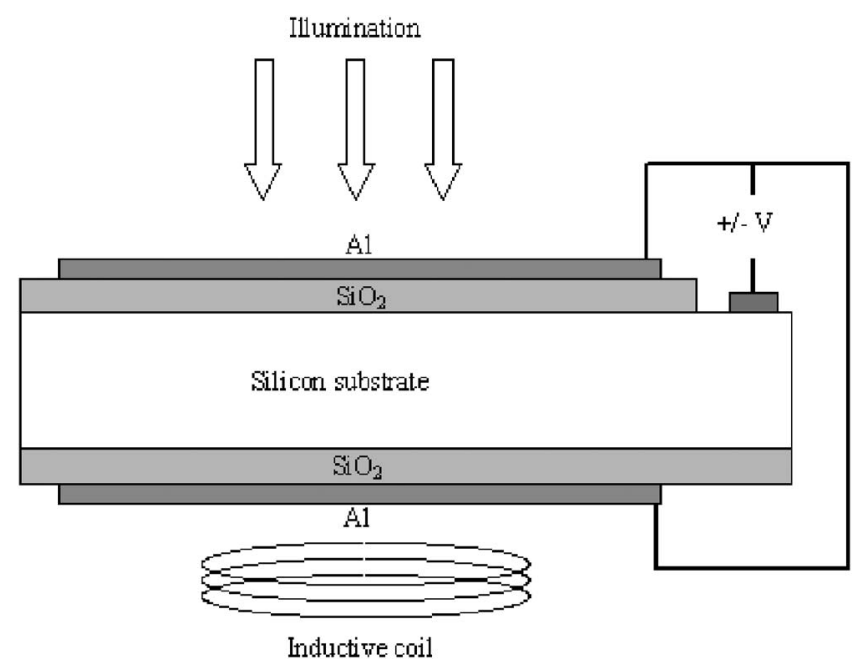

FIG. 1. Schematic setup for the transient lifetime measurement using a MOS structure to allow charging of the surfaces.

In high level injection, the instantaneous decay time $\tau_{\text {inst }}$ is $^{6}$

$$
1 / \tau_{\text {inst }}=1 / \tau_{\text {bulk,hli }}+\left(2 J_{0 e} n\right) /\left(q W n_{1}^{2}\right),
$$

where $\tau_{\text {bulk,hli }}$ is the high level injection lifetime of the wafer bulk, $n$ is the photogenerated excess carrier density in the wafer bulk, $W$ is the wafer thickness, $n_{i}$ is the intrinsic carrier concentration, $J_{\mathrm{oe}}$ is the emitter dark saturation current density, and $q$ the electronic charge. The value of $J_{\mathrm{oe}}$ can be determined from the slope of $1 / \tau_{\text {inst }}$ vs $n$ after Auger recombination has been accounted for. $J_{\mathrm{oe}}$ is an indicator of the degree of surface passivation and a parameter of direct technological importance for photovoltaics. Measurement of $J_{\mathrm{oe}}$, rather than the effective surface recombination velocity, has the advantage that it allows the unambiguous separation of surface and bulk recombinations. Both $\tau_{\text {inst }}$ and $J_{\text {oe }}$ were measured at an injection level of $4 \times 10^{15} \mathrm{~cm}^{-3}$. For all measurements reported here, $n$ is uniform throughout the wafer due to the high bulk lifetime and low surface recombination rates.

Figure 2 shows the measured effective lifetime as a function of applied bias voltage $V_{\text {app }}$ in the range $-11-+11 \mathrm{~V}$. The corresponding applied charge density varies from -4.7 $\times 10^{12} \mathrm{~cm}^{3}$ to $+4.7 \times 10^{12} \mathrm{~cm}^{-3}$. The curve displays the characteristic "U" shape, with a minimum in the effective lifetime around $-1.2 \mathrm{~V}$. The work function difference of the $\mathrm{Al}-\mathrm{SiO}_{2}-\mathrm{Si}$ system for the sample used is $\sim-0.8 \mathrm{~V}$. $^{8}$ If we

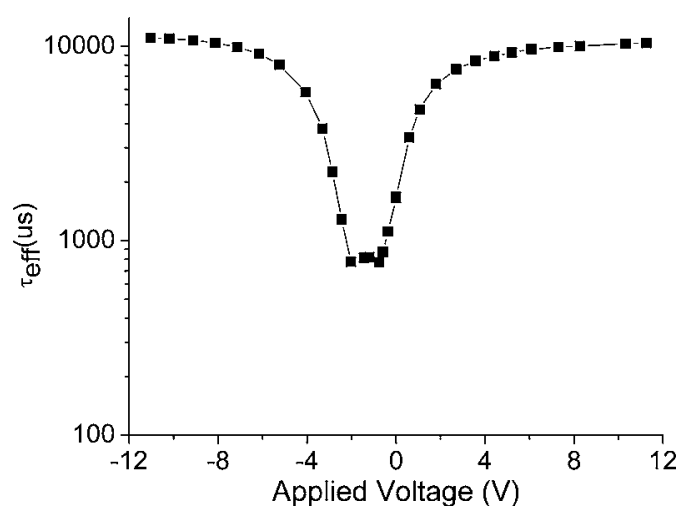

FIG. 2. Instantaneous effective lifetime $\tau_{\text {inst }}$ measured at an injection level of $4 \times 10^{15} \mathrm{~cm}^{-3}$ as a function of applied voltage.

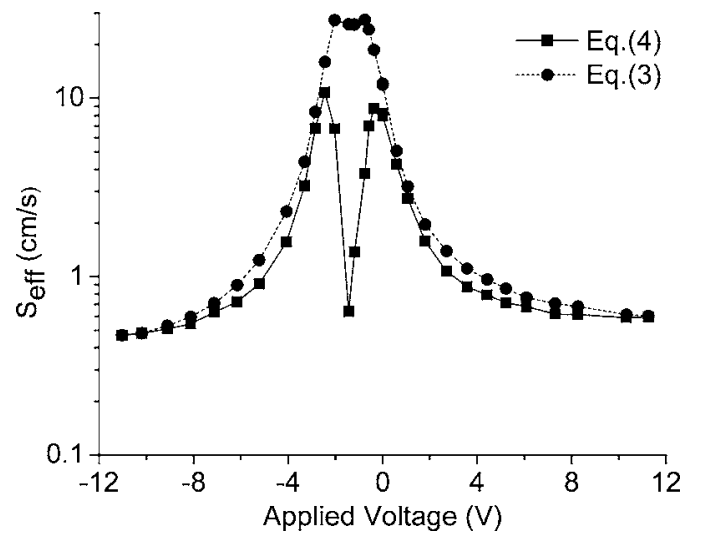

FIG. 3. Emitter saturation current $J_{\mathrm{oe}}$ measured at an injection level of 4 $\times 10^{15} \mathrm{~cm}^{-3}$ as a function of applied voltage.

assume that the minimum in the effective lifetime curve corresponds to a depleted $\mathrm{Si}-\mathrm{SiO}_{2}$ interface, we can estimate the effective charge density due to fixed oxide charges to be $+2 \times 10^{11} \mathrm{~cm}^{-3}$.

Figure 3 shows the measured values for the emitter saturation current density $J_{\mathrm{oe}}$ on the same sample. A valid measurement of $J_{\mathrm{oe}}$ can only be made under the conditions that (i) the surface of the sample is in low level injection and (ii) the bulk of the sample is in high level injection (as a general rule, delta $n$ should be at least equal to $10 n_{0}$ ). Both conditions must be met over the entire injection level range used to extract the value of $J_{\mathrm{oe}}$. Condition (ii) is met over the entire range of $V_{\text {app }}$, but condition (i) clearly is not met for low values of $V_{\text {app }}$. Calculation of the surface doping concentration as a function of applied charge density shows that the electron or hole concentration exceeds $10^{18} \mathrm{~cm}^{-3}$ for applied voltages of greater than $0.3 \mathrm{~V}$ or less than $-2.7 \mathrm{~V}$, respectively. Note that while $J_{\mathrm{oe}}$ usually includes contributions from both the bulk and the surface regions due to the extremely sharp carrier profile in the silicon surface region resulting from the applied charge, the contribution from the silicon bulk is negligible in this case.

The parameter more usually measured in relation to surface recombination is the effective surface recombination velocity $S_{\text {eff }}$, defined by

$$
S_{\text {eff }}=U / n,
$$

where $U$ is the surface recombination rate per unit area. $S_{\text {eff }}$ is typically determined from the following equation, valid for relatively low values of $S_{\text {eff: }}$ :

$$
1 / \tau_{\text {inst }}=1 / \tau_{\text {bulk }}+2 S_{\text {eff }} / W \text {. }
$$

Estimating $S_{\text {eff }}$ therefore requires the bulk lifetime, $\tau_{\text {bulk }}$ to be known. Since $\tau_{\text {bulk }}$ cannot be separately measured, an assumed value must be used which makes it difficult to accurately determine low values of $S_{\text {eff. }}$. However, the measurement of $J_{\mathrm{oe}}$ allows accurate determination of $\tau_{\text {bulk. }} . S_{\text {eff }}$ can be calculated from measured $J_{\text {oe }}$ values from the relation

$$
S_{\text {eff }}=J_{0}\left(p_{0}+n\right) /\left(q n_{i}^{2}\right),
$$

where $p_{0}$ is the hole concentration in thermal equilibrium $\left(10^{14} \mathrm{~cm}^{-3}\right)$.

We use Eq. (4) to determine $S_{\text {eff }}$ at the injection level of $4 \times 10^{15} \mathrm{~cm}^{-3}$ for the data point from Fig. 2 with the highest measured lifetime, which was obtained with $V_{\text {app }}=-11 \mathrm{~V}$. This gives a value of $\tau_{\text {bulk }}$ of $14.4 \mathrm{~ms}$. Substitution of this license or copyright; see http://apl.aip.org/about/rights_and_permissions 


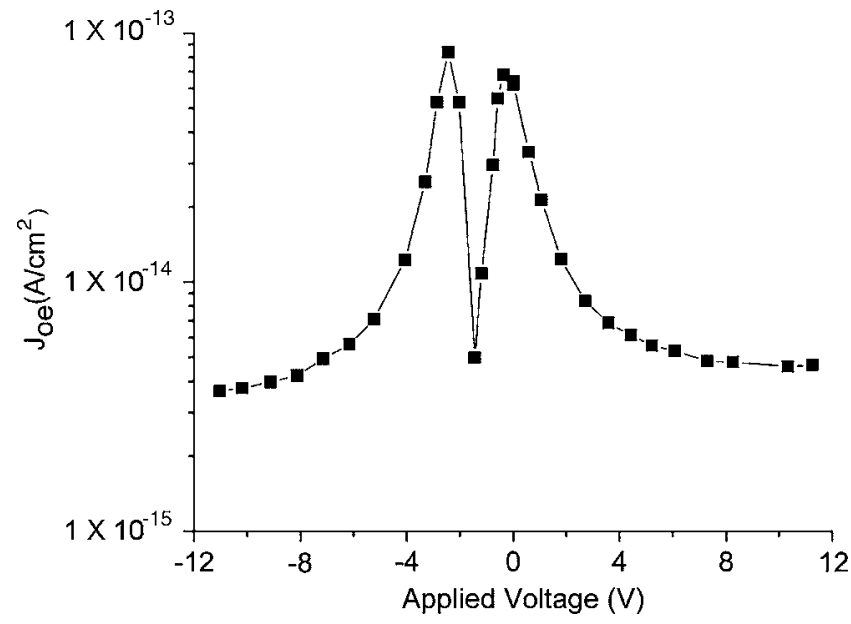

FIG. 4. Effective surface recombination velocity $S_{\text {eff }}$ as a function of application voltage, calculated from Eq. (4) (squares) and Eq. (3) (circles).

value into Eq. (3) then allows determination of $S_{\text {eff }}$ over the entire range of applied voltages. Figure 4 shows the variation of $S_{\text {eff }}$ as a function of $V_{\text {app }}$ for $n=4 \times 10^{15} \mathrm{~cm}^{-3}$. For comparison, the values of $S_{\text {eff }}$ extracted from the application of Eq. (4) to the $J_{\text {oe }}$ data of Fig. 3 are also shown. There is a good agreement between the two curves, except for the range of voltages where the condition that the surface be in low level injection is not met.

The lowest measured $J_{\mathrm{oe}}$ value from Fig. 3 is $1.8 \mathrm{fA} / \mathrm{cm}^{2}$ per surface, for $V_{\text {app }}=-11 \mathrm{~V}$. Such a value of $J_{\mathrm{oe}}$ on both surfaces would impose a limit on the open circuit voltage of a solar cell of $780 \mathrm{mV}$, assuming a short circuit current of $40 \mathrm{~mA} / \mathrm{cm}^{2}$. From Eq. (4), the corresponding value of $S_{\text {eff }}$ at low injection levels (below $10^{13} \mathrm{~cm}^{-3}$ ) is $0.012 \mathrm{~cm} \mathrm{~s}^{-1}$. This value was obtained on a sample which received a standard forming gas annealing. Since it is known that the so-called alneals result in better surface passivation, ${ }^{9}$ particularly for positively charged surfaces, it is likely that even lower $J_{\mathrm{oe}}$ values can be achieved with such treatments.

The authors would like to thank Keith McIntosh, Hao Jin, and Andres Cuevas for many useful discussions. Financial support from the Australian Research Council is gratefully acknowledged.

${ }^{1}$ M. Schoefthaler, R. Brendel, G. Langguth, and J. H. Werner, Proceedings of the First World Conference on Photovoltaic Energy Conversion, Hawaii, 5-9 December 1994, p. 1509.

${ }^{2}$ S. Dauwe, J. Schmidt, A. Metz, and R. Hezel, Proceedings of the 29th IEEE Photovoltaic Specialists Conference, New Orleans, LA, 17-24 May 2002, p. 162.

${ }^{3}$ J. Zhao, J. Schmidt, A. Wang, G. Zhang, B. S. Richards, and M. A. Green, Proceedings of the Third World Conference on Photovoltaic Energy Conversion, Osaka, Japan, 12-16 May 2003, p. 293.

${ }^{4}$ E. Suzuki, H. Takato, K. Ishii, and Y. Hayashi, Jpn. J. Appl. Phys., Part 2 29, L2300 (1990).

${ }^{5}$ W. Kern and D. A. Puotinen, RCA Rev. 31, 187 (1970).

${ }^{6}$ D. E. Kane and R. M. Swanson, Proceedings of the 18th IEEE Photovoltaic Specialists Conference, Las Vegas, NV, 21-26 October 1985, p. 578.

${ }^{7}$ E. Yablonovitch and T. G. Gmitter, Solid-State Electron. 35, 261 (1992).

${ }^{8}$ W. M. Werner, Solid-State Electron. 17, 769 (1974).

${ }^{9}$ M. A. Green, Silicon Solar Cells: Advanced Principles and Practice (Centre for Photovoltaic Devices and Systems, University of NSW, Sydney, Australia, 1995), p. 169. 